Scanning electron acoustic microscopy of indium-doped semi-insulating GaAs

This article has been downloaded from IOPscience. Please scroll down to see the full text article.

1993 Semicond. Sci. Technol. 8320

(http://iopscience.iop.org/0268-1242/8/3/002)

View the table of contents for this issue, or go to the journal homepage for more

Download details:

IP Address: 147.96.14.16

The article was downloaded on 22/02/2013 at 18:31

Please note that terms and conditions apply. 


\title{
Scanning electron acoustic microscopy of indium-doped semi-insulating GaAs
}

\author{
B Méndez and J Piqueras \\ Departamento de Física de Materiales, Facultad de Físicas, Universidad \\ Complutense, 28040 Madrid, Spain
}

Received 28 October 1992, in final form 17 December 1992, accepted for publication 5 January 1993

\begin{abstract}
Dislocation lines in $\{110\}$ sections of In-doped GaAs are imaged by scanning electron acoustic microscopy (SEAM). Cathodoluminescence measurements show the presence of a high indium concentration at dislocations. it is proposed that the semi-insulating property of the sample and dislocation decoration contribute to the SEAM contrast.
\end{abstract}

\section{Introduction}

Doping LEC-GaAs with a few per cent of indium can reduce the dislocation density by several orders of mag-. nitude [1]. Semi-insulating indium-doped GaAs is therefore of interest as a homogeneous substrate suitable for device fabrication. Several methods have been used in recent years to observe and characterize the defects present in In-doped LEC GaAs. In (110) specimens, straight $\langle 100\rangle$ dislocations decorated by precipitates as well as doping striations have been observed by $\mathrm{x}$-ray topography and infrared absorption $[2,3]$. Scanning electron acoustic microscopy (SEAM) has been used in recent years to characterize compound semiconductors. In the case of GaAs, SEAM has been found [4] to be much more useful for characterizing semi-insulating material than n-type material. SEAM images of semi-insulating undoped and $\mathrm{Cr}$-doped GaAs revealed $[4,5]$ the cellular structure in these materials while single dislocations were not imaged. Due to the low dislocation density and the semi-insulating properties of GaAs:In, this material appears to be suitable for further study of the SEAM capability in GaAs characterization and in particular for revealing single dislocations under specific experimental conditions. In the present work SEAM has been used to observe $\{110\}$-oriented GaAs:In. The observations were complemented by SEM cathodoluminescence (CL) methods.

\section{Experimental methods}

A $\{110\}$ sample of indium-doped LEC semi-insulating GaAs was used. The sample had a thickness of $1 \mathrm{~mm}$ and polished faces. SEAM measurements were performed in a Cambridge \$4-10 or a Hitachi 2500 scanning electron microscope. In order to get additional information on the defects, CL was measured in the same instruments. The $\mathrm{CL}$ experimental arrangement has been described previously $[5,6]$. The SEAM system used in this work has also been described [7]. Basically it consists of a chopping system with a pair of condenser plates and beam blanking electronics to create a periodically modulated beam with frequencies in the range $40-240 \mathrm{kHz}$. The acoustic signal is detected by a piezoelectric ceramic transducer on which the sample is clamped.

\section{Results and discussion}

Dislocations with a certain structure along their lines are observed in the SEAM and $\mathrm{CL}$ images (figure 1). The structure, part of which is marked with arrows in figure 1 , is probably related to the decoration of the dislocation by impurities and precipitates. The presence of precipitates spaced several microns along dislocation lines in GaAs:In has been described by several authors (for instance in $[2,8]$ ) and seems to agree with the present SEAM observations. It appears then that SEAM allows the observation of dislocation lines in GaAs: In as well as decorating particles. This is a consequence of the particular experimental conditions of this work. The main generation mechanisms of the electron acoustic signal in semiconductors are piezoelectric and excess carrier coupling [9]. In [4] it has been reported that dislocations in n-type GaAs, readily observable by $\mathrm{CL}$, are not visible in the SEAM image due to the high carrier concentration that screens the piezoelectric contribution to the acoustic signal. Only cellular structure and possibly dislocation emergency points are imaged by SEAM in semi-insulating material. It is suggested that the semi-insulating property of the samples used here together with the strong decoration of dislocations by 

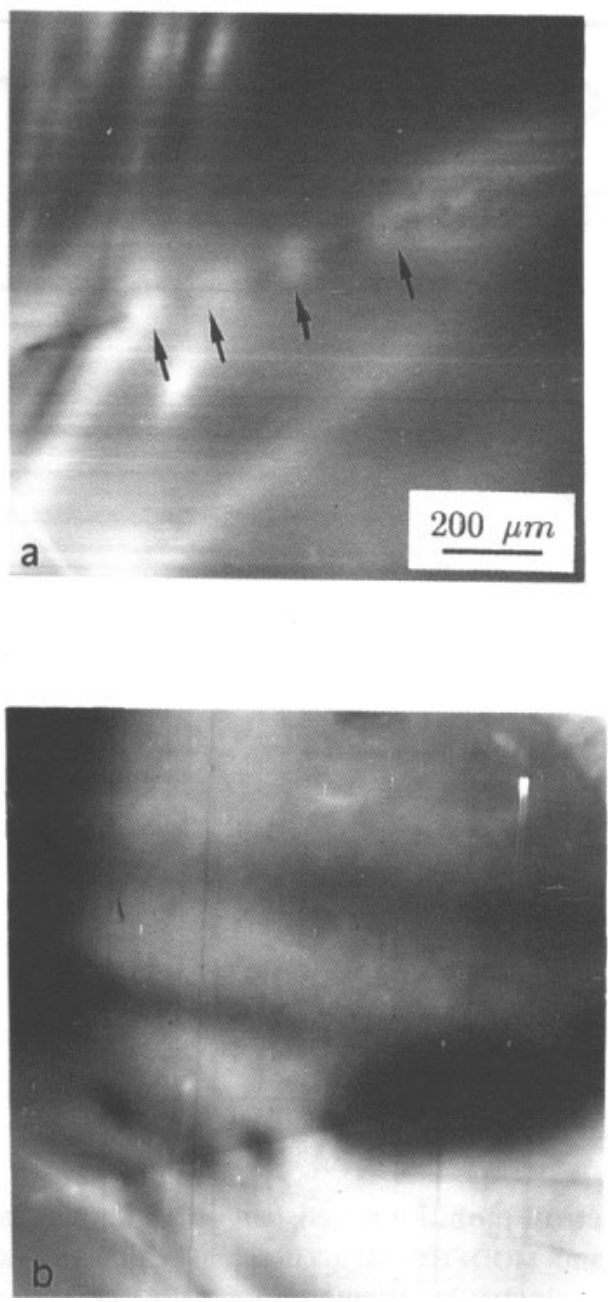

Figure 1. (a) SEAM and (b) a images of the same dislocation line of a GaAs:In sample. The SEAM image was recorded at room temperature, with a beam energy of $30 \mathrm{keV}$ and a chopping frequency of $100 \mathrm{kHz}$. Arrows show decoration features along the dislocation line.

indium impurities allow dislocations to be imaged by SEAM. In [4] it has been suggested that the reason for strong SEAM defect contrast of semi-insulating GaAs is related to a piezoelectric signal generation mechanism. In this mechanism the electron beam injection creates a varying electric field in the piezoelectric material, and consequently elastic waves are generated.

A possible contribution to the observed SEAM contrast, besides the piezoelectric contribution, is the effect of a high indium concentration at the dislocation. Bresse and Papadopoulo $[10,11]$ have shown that the electron acoustic signal varies with the doping level in GaAs and observed doping striations in heavily $\left(10^{20}\right.$ atoms $\mathrm{cm}^{-3}$ ) Be-doped GaAs. This effect was attributed to the impurity-induced variation of the thermal conductivity of the semiconductor. This suggests that the dislocation contrast could be enhanced by a high indium decoration. In order to investigate this possibility $\mathrm{CL}$ spectra have been recorded at different points of the sample. Figure 2 shows the CL spectra at the dislocation line, in its proximity and in a dislocation-free region. The different points were determined from the $c \mathrm{im}$ -

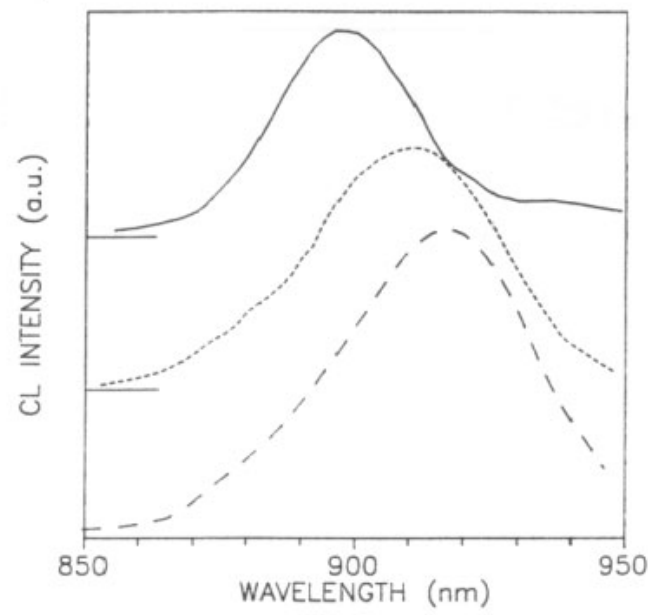

Figure 2 a spectra from different positions in the sample. The full curve corresponds to a spectrum from a dislocation-free region; the long and short broken curves are spectra recorded at the dislocation line and in its proximity respectively.

age. The peaks of the spectra appear with a relative shift which is attributed to the different local indium concentration. Kirillov et al [12] have reported that the indium content determines the position of the luminescence band maxima. Taking into account this observation, the spectra of figure 2 reveal a higher (about a factor 2) indium concentration at the dislocation than in a region that is distant from dislocation lines. This result supports the possibility that indium decoration contributes to the dislocation SEAM contrast.

\section{Acknowledgments}

This work was supported by the Volkswagen Foundation and by DGICYT (Project PB 90-1017).

\section{Reterences}

[1] Jacob G, Farges J P, Schemali C, Duseaux M, Hallais J, Bartels W J and Roksnoer P J $1982 \mathrm{~J}$. Crystal Growth 57245

[2] Stirland D J, Hart D G, Grant I, Brozel M R and Clark S 1987 Microscopy of Semiconducting Materials 1987 (Inst. Phys. Conf. Ser. 87) p 269

[3] Kidd P, Booker G R and Stirland D J 1987 Microscopy of Semiconducting Materials 1987 (Inst. Phys. Conf. Ser. 87) p 275

[4] Méndez B and Piqueras J 1992 Appl. Phys. Lett. 60 1357

[5] Méndez B and Piqueras J 1989 Microscopy of Semiconducting Materials 1989 (Inst. Phys. Conf. Ser. IO0) p 789

[6] Méndez B and Piqueras J 1992 J. Appl. Phys. 692776

[7] Urchulutegui $M$, Piqueras $J$ and Uopis J $1989 \mathrm{~J}$. Appl Phys. 652667

[8] Yonenaga I and Sumino K $1987 \mathrm{~J}$. Appl Phys, 621212

[9] Kultscher N and Balk L J 1986 Scanning Electron Microscopy I p 33

[10] Bresse J F and Papadopoulo A C 1988 J. Appl. Phys. 64 98

[11] Bresse J F and Papadopoulo A C 1987 Appl. Phys. Lett. 51183

[12] Kirillov D, Vichr M and Powell R A 1987 Appl. Phys, Lett. $\mathbf{5 0} 262$ 